\title{
A Frameshift Variant in KIAA0825 Causes Postaxial Polydactyly
}

\author{
Muhammad Bilal Wasim Ahmad \\ Department of Biochemistry, Faculty of Biological Sciences, Quaid-i-Azam University, Islamabad, Pakistan
}

\section{Keywords}

Postaxial polydactyly $\cdot$ KIAA0825 - Frameshift variant

\begin{abstract}
Postaxial polydactyly (PAP) is characterized by counterproductive 5th digit (pinky finger) duplication on hands and/or feet which often leads to functional complications. To date, at least 11 genes involved in causing various types of nonsyndromic polydactylies have been reported. In the present study, a consanguineous family of Sindhi origin with a segregating nonsyndromic form of PAP in an autosomal recessive manner was clinically and genetically evaluated. Genotyping, using polymorphic microsatellite markers, established linkage in the family on chromosome $5 q 15$ harboring the KIAA0825 gene (MIM 617266). Sequence analysis of the gene revealed a novel frameshift variant leading to a premature stop codon [c.143delG, p.(Cys48Serfs*28)]. This is only the 4th novel variant in the KIAA0825 gene that leads to PAP type A10 (PAPA10) (MIM 618498). Identification of variants in the PAP causative gene will support the diagnosis of patients with limb malformations in the Pakistani population.
\end{abstract}

(c) 2020 S. Karger AG, Basel

$\begin{aligned} & \text { karger@karger.com } \\ & \text { www.karger.com/msy }\end{aligned}$
Karger $\%$

\section{Introduction}

Development of the limbs is associated with various cellular signaling pathways that are still not fully decoded. A study of human hereditary limb malformations has identified few genes responsible for causing various types of limb defects.

Polydactyly is an anomaly in humans resulting in supernumerary fingers and/or toes on one or both hands/ feet. The extra digit(s) present on the little finger side on the hands or the 5th toe on the feet is known as postaxial polydactyly (PAP), on the thumb side on the hands or the big toe on the feet as preaxial, and involving middle digits in hands and feet as central polydactyly. Hands are more affected than feet, and it occurs in 1:1,000 births in the general population [Biesecker, 2006; Umair et al., 2018]. The syndromic form of polydactyly is much less common than nonsyndromic polydactyly. Malik et al. [2014] reported that $52 \%$ of nonsyndromic polydactylies are postaxial in the Pakistani population.

Nonsyndromic polydactyly has been divided into 2 types, including PAP type A (PAP-A) with a well-developed extra 5th digit and PAP type $\mathrm{B}$, characterized by incomplete development of an extra digit attached to the 
Table 1. List of candidate genes used for linkage analysis in the family

\begin{tabular}{lllll}
\hline Disease & Gene & Inheritance & Locus & OMIM \\
\hline Postaxial polydactyly & GLI3 & AD & $7 \mathrm{p} 14.1$ & 174700 \\
& GLI1 & $\mathrm{AR}$ & $12 \mathrm{q} 13.3$ & 165220 \\
& ZNF141 & $\mathrm{AR}$ & $4 \mathrm{p} 16.3$ & 615226 \\
& IQCE & $\mathrm{AR}$ & $7 \mathrm{p} 22.3$ & 617642 \\
& FAM92A & $\mathrm{AR}$ & $8 \mathrm{q} 22.1$ & 617273 \\
& KIAA0825 & $\mathrm{AR}$ & $5 \mathrm{q} 15$ & 617266 \\
\hline
\end{tabular}

$\mathrm{AR}$, autosomal recessive; $\mathrm{AD}$, autosomal dominant

5th finger or toe [Kalsoom et al., 2013]. To date, 6 genes (GLI3, GLI1, ZNF141, IQCE, FAM92A, KIAA0825) for postaxial, 3 (GLI3, SHH, STKLD1) for preaxial, and 2 (MIPOL1, PITX) for complex forms of nonsyndromic polydactylies have been reported.

In the present study we have investigated a consanguineous family of Sindhi origin showing PAP-related phenotypes. Sequence analysis revealed a novel frameshift variant [c.143delG, p.(Cys48Serfs*28)] in the recently reported KIAA0825 gene.

\section{Materials and Methods}

\section{Human Subjects}

A consanguineous Sindhi speaking family with segregating PAP-A in an autosomal recessive pattern was ascertained from a remote area in the Sindh province of Pakistan.

\section{Extraction of Genomic DNA and Genotyping}

Peripheral blood samples were collected in EDTA-containing vacutainer sets from 5 members including 2 affected (IV-2, IV-3) and 3 unaffected (III-1, IV-1, IV-4) subjects. Extraction of genomic DNA was carried out using a standard phenol-chloroform method. Linkage to candidate genes in the family was tested by typing highly polymorphic microsatellite markers mapped in the vicinity of the genes involved in nonsyndromic PAP (Table 1). Haplotypes were constructed to establish linkage in the family.

\section{Sequencing of KIAA0825}

Primers for PCR amplification of exons and splice junctions of the KIAA0825 gene (NM_173665) were designed using the online available Primer3 tool (http://bioinfo.ut.ee/primer3--0.4.0/). The thermal cycling conditions used were $95^{\circ} \mathrm{C}$ for $5 \mathrm{~min}$, followed by 35 cycles of $95^{\circ} \mathrm{C}$ for $40 \mathrm{~s}, 56^{\circ} \mathrm{C}$ for $40 \mathrm{~s}$, and $72^{\circ} \mathrm{C}$ for $60 \mathrm{~s}$ with a final extension at $72^{\circ} \mathrm{C}$ for $10 \mathrm{~min}$. The PCR-amplified products were purified with a commercially available kit (Axygen). Sanger sequencing was performed bidirectionally using a standard protocol [Aziz et al., 2014]. Sequencing results were aligned, and the variant was examined using BIOEDIT sequence alignment editor version 6.0.7 (Ibis Biosciences). Conservation of the amino acid, altered in the variant, was determined in several different species using HomoloGene-NCBI. Pathogenicity of the variant was analyzed using different online available tools, including MutationTaster, PolyPhen-2, FATHMM-MKL, Provean, SIFT, ExAC, and gnomAD.

\section{Isolation of RNA and cDNA Synthesis}

For isolation of total RNA, 3 mL peripheral blood samples were collected in Tempus Blood RNA Tubes (Applied Biosystems) from 2 affected and 2 unaffected members of the family. Total RNA isolation and cDNA preparation was performed as previously described [Aziz et al., 2014].

\section{Results}

\section{Clinical Features}

A consanguineous Sindhi speaking family with segregating nonsyndromic PAP-A in an autosomal recessive manner was investigated in the present study (Fig. 1A). The family had 2 affected individuals (IV-2, 40 years; IV3,19 years). Affected individual IV-2 presented a bilateral well-developed duplication of the 5 th digit on hands and feet, which was compatible with PAP-A. Radiographs revealed a diphalangeal 6 th finger on both hands. The right foot of patient IV-2 showed a misshaped medial cuneiform bone, overlapping mildly the $2 \mathrm{nd}$, $3 \mathrm{rd}$, and 4 th metatarsals. A bifid distal head of the 5th metatarsal was observed, and mild cutaneous syndactyly was present in the feet (Fig. 1B-E).

Physical examination and X-rays of the other affected individual (IV-3) demonstrated unilateral PAP-A on the left hand with an additional digit at the fibular/postaxial side of the hand. The right hand was not affected. An abnormality was also recognized in the carpal bones as trapezium, trapezoid, pisiform, triquetrum, and lunate were missing in both hands. He had a well-developed duplication of the 5 th digit on the feet, which was also clearly seen on X-rays. A broad 5th metatarsal was observed in the right foot of subject IV-3 (Fig. 1F-I). Abnormalities involving cranium, face, heart, teeth, and hearing were not observed in both affected members. Developmental delay was not noticed, and they had a normal skull, eyesight, nails, height, and weight.

\section{Genotyping and Sanger Sequencing}

Analysis of the haplotypes, constructed by typing microsatellite markers, established linkage in the family on chromosome 5q15 harboring the causative KIAA0825 gene (NM_173665). Therefore, the same gene was Sangersequenced in all available affected and unaffected members of the family, and sequence analysis revealed a novel 


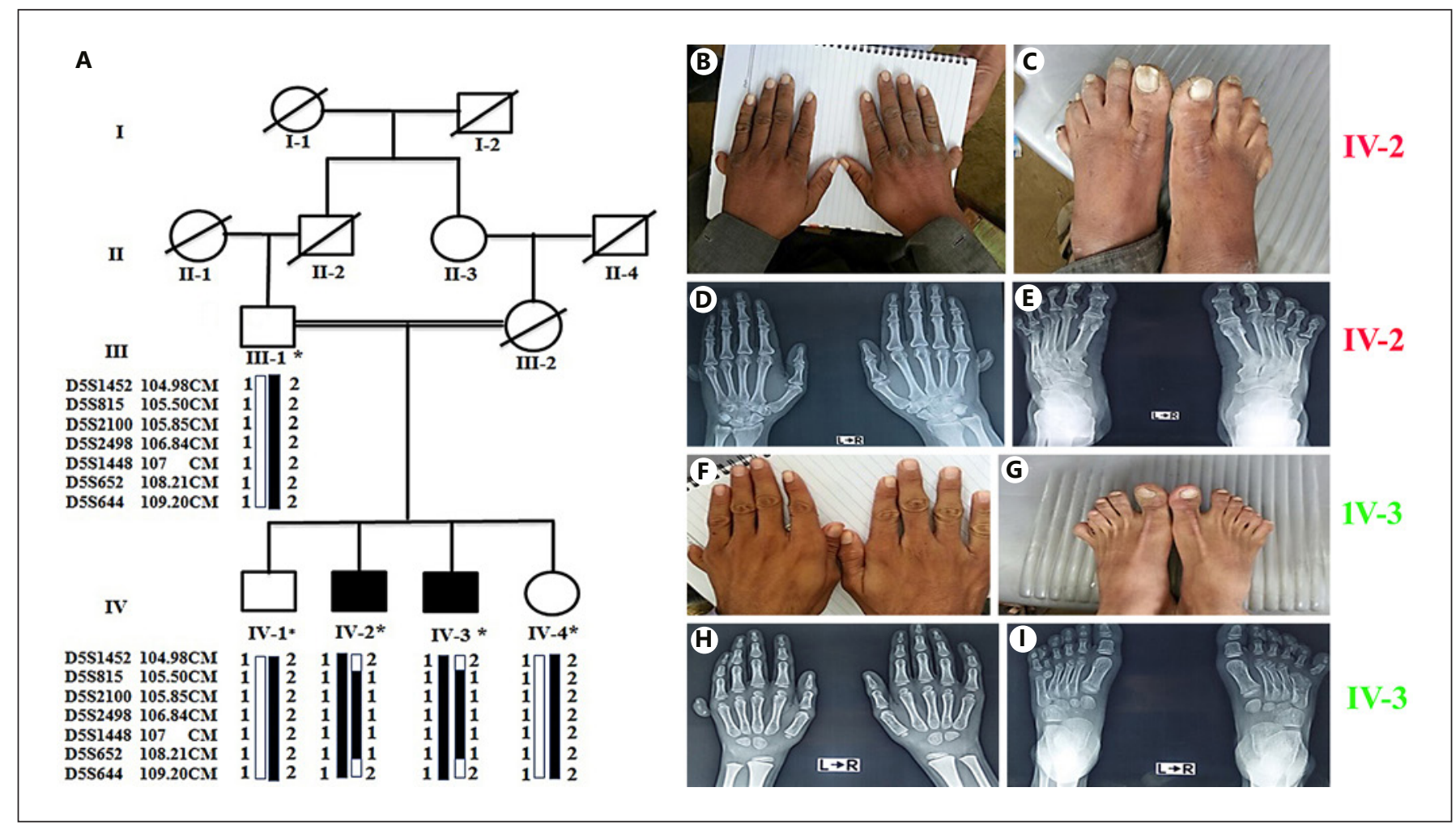

Fig. 1. A Pedigree of the family with segregating PAP in an autosomal recessive manner. Circles and squares represent females and males, respectively. Clear symbols represent unaffected members, while filled symbols show affected members. Double lines are indicative of consanguineous unions. The individual numbers labeled with asterisks denote the samples which were available for this study. A cross line on the symbol indicates a deceased individual. B, C Hands and feet of affected individual IV-2 with PAP-A. D, E Radiograph of affected individual IV-2 showing PAP on hands and feet. F, G Hands and feet of affected individual IV-3 representing PAP-A. H, I X-rays of affected individual IV-3 exhibiting PAP on the left hand only and PAP on the feet. homozygous single base pair deletion [c.143delG, p.(Cys48Serfs*28)] in exon 4 of the gene. The deletion led to a frameshift and generated a premature stop codon $28 \mathrm{ami}$ no acids downstream of the variant position (Fig. 2B-D). The identified variant was found conserved in both isoforms of the KIAA0825 protein. The UCSC coordinates for the identified variant was chr:5g.93859792_93859792delC. Human GRCh37 genome assembly was used as a reference. The variant was not found in 110 in-house exomes, 1,000 genome project, ExAC, and gnom $A D$, and it was predicted to be deleterious by online available bioinformatics tools, including MutationTaster, PolyPhen-2, FATHMM-MKL, Provean, and SIFT. Analysis by HomoloGene-NCBI revealed that the mutated amino acid cysteine was conserved across different species (Fig. 2E). Reverse-transcriptase PCR using RNA extracted from blood of an affected individual failed to amplify the KIAA0825 mRNA. However, it was successfully amplified from unaffected individuals (Fig. 2F). For PCR amplification of KIAA0825 cDNA, the primer set forward $5^{\prime}$ CATTTCCTGGAGACTTGGAGTTTG $3^{\prime}$ and reverse $5^{\prime}$ TGGGAATGAAACGCTGCTGT 3' was used.

\section{Discussion}

Abnormalities of human hands and feet occur frequently in the general population either as an isolated anomaly or as a part of complex syndromes. The development of human limbs is regulated by a series of complex cellular pathways, including hedgehog, WNT, and bone morphogenetic proteins (BMP). Deficiency of any regulator in such pathways leads to various types of limb deformities [Biesecker, 2011; Stewart et al., 2017]. Polydactyly is one of such deformities that results due to defects 


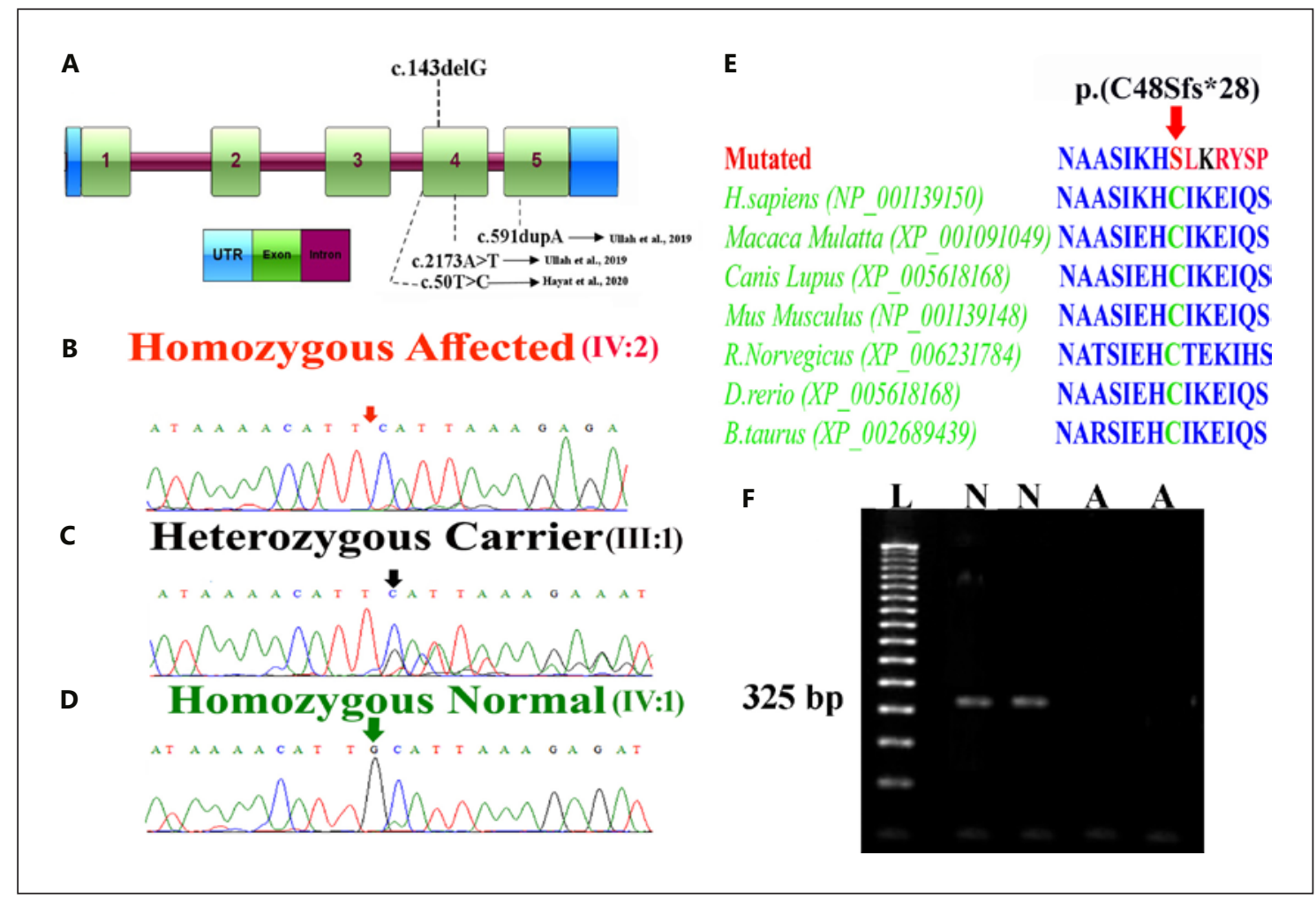

Fig. 2. A KiAA0825 gene structure showing exons and position of the deletion c.143delG. B-D Sequencing chromatograms in homozygous affected, heterozygous carrier, and homozygous unaffected individuals. Arrows represent the mutation. E Conservation of the mutated amino acid across different species. $\mathbf{F}$ PCR amplification of KIAA0825 cDNA in unaffected and affected individuals. L, DNA ladder; N, normal members; A, affected members of the family.

in the anterior-posterior patterning of limb development [Ullah et al., 2019].

To date, at least 6 genes (GLI1, GLI3, ZNF141, IQCE, FAM92A, KIAA0825) causing nonsyndromic PAP have been reported, and an involvement of the KIAA0825 gene was recently shown in 2 families of Pakistani origin [Ullah et al., 2019]. A homozygous knock-out mouse $\left(2210408 I_{21}\right.$ Rik $\left.^{\text {tm } 1 b(E U C O M M) W t s i}\right)$ revealed several skeletal anomalies affecting growth, body size, and decrease in bone mineral density [Koscielny et al., 2014]. To date, only 3 variants [p.(Gln198Thrfs*21), p.(Lys725*), and p.(Leu17Ser)] in the KIAA0825 gene causing PAP have been reported [Ullah et al., 2019; Hayat et al., 2020]. The frameshift [c.143delG, p.(Cys48Serfs*28)] identified in our family is the 4th variant, and the clinical features observed were mostly comparable to those carrying the previously reported variants in the KIAA0825 gene.

KIAA 0825 has 2 isoforms, including a long form containing 1,275 amino acids and a short form with 324 ami- no acids. It is highly likely that the frameshift variant p.(Cys48Serfs*28) identified in our family affects both isoforms which leads to degradation of KIAA0825 RNA through nonsense-mediated mRNA decay. RT-PCR analysis validated the same effect.

In conclusion, we have reported a 4 th novel variant in KIAA0825 causing autosomal PAP-A. This study not only expands the mutation spectrum of KIAA0825, but it will also facilitate the screening of patients carrying polydactyly-related phenotypes.

\section{Acknowledgements}

The authors are highly obliged to the family members for their participation in the study. M.B. was supported by an indigenous $\mathrm{PhD}$ fellowship from Higher Education Commission (HEC), Islamabad, Pakistan. 


\section{Statement of Ethics}

The study was ethically conducted in accordance with the World Medical Association Declaration of Helsinki. It was approved by the Institutional Review Board (IRB) of Quaid-i-Azam University, Islamabad, Pakistan. Informed written consent to perform the study and publish the research data was obtained from guardians of theaffected members of the family.

\section{Conflict of Interest Statement}

The authors have no conflicts of interest to declare.

\section{Funding Source}

Funds were not provided by any government/nongovernment organization.

\section{Author Contributions}

M.B. drafted the manuscript, collected the samples, performed experiments, and analyzed the data. W.A. analyzed clinical and experimental data, provided funds for the study, and edited the manuscript.

\section{References}

Aziz A, Irfanullah I, Khan S, Zimri FK, Muhammad N, Rashid S, et al. Novel homozygous mutations in the WNT10B gene underlying autosomal recessive split hand/foot malformation in three consanguineous families. Gene. 2014;534(2):265-71.

Biesecker LG. Polydactyly: how many disorders and how many genes: 2010 update. Dev Dyn. 2011;240(5):931-42.

Hayat M, Umair M, Abbas S, Rauf A, Ahmad F, Ullah S, et al. Identification of novel biallelic missense variant in the KIAA0825 underlies postaxial polydactyly type A. Genomics. 2020; 19:30622-6.
Kalsoom UE, Klopocki E, Wasif N, Tariq M, Khan $\mathrm{S}$, Hecht $\mathrm{J}$, et al. Whole exome sequencing identified a novel zinc-finger gene ZNF141 associated with autosomal recessive postaxial polydactyly type A. J Med Genet. 2013;50(1): 47-53.

Koscielny G, Yaikhom G, Iyer V, Meehan TF, Morgan $\mathrm{H}$, Atienza-Herrero J, et al. The international mouse phenotyping consortium web portal, a unified point of access for knockout mice and related phenotyping data. Nucleic Acids Res. 2014;42(Database issue):D802-9.
Malik S, Ullah S, Afzal M, Lal K, Haque S. Clinical and descriptive genetic study of polydactyly: a Pakistani experience of 313 cases. Clin Genet. 2014;85(5):482-6.

Stewart TA, Bhat R, Newman SA. The evolutionary origin of digit patterning. Evodevo. 2017; $8: 21$.

Ullah I, Kakar N, Schrauwen I, Hussain S, Chakchouk I, Liaqat K, et al. Variants in KIAA0825 underlie autosomal recessive postaxial polydactyly. Hum Genet. 2019;138(6):593-600.

Umair M, Ahmad F, Bilal M, Ahmad W, Alfadhel M. Clinical genetics of polydactyly: an updated review. Front Genet. 2018;9:447. 